\title{
Spectrum Emitted by a Trapped Electron
}

\author{
Pietro Paolo Corso, Emilio Fiordilino, Gianfranco Orlando, Franco Persico \\ Dipartimento di Scienze Fisiche e Astronomiche \\ Università di Palermo, Via Archirafi 36 \\ 90123 Palermo, Italy \\ e-mail: fiordili@fisica.unipa.it (E. Fiordilino)
}

(Received: June 26, 2006)

\begin{abstract}
We study the behaviour of a homonuclear molecule driven by an intense laser field. Newton's laws are used to describe the dynamics of nuclei while the quantal approach is reserved to the study of the electron. It is observed that the nuclei can oscillate or dissociate according to the degree of ionization of the molecule. In case of low ionization rate it is shown that great amount of information can be obtained by using the simplified approaches of fixed nuclei and of two-state approximation. Under suitable conditions the electron wave function spends a long time localized around one nucleus. The harmonic generation of the molecule is studied and seen to contain even harmonics.
\end{abstract}

\section{Introduction}

Atoms and molecules acted upon by a laser of frequency $\omega_{L}$ emit a radiation field whose spectrum can be resolved in a wide plateau of lines harmonic of $\omega_{L}$ followed by a rapid decrease of the emission (cutoff). The radiation stems from the acceleration experienced by the electronic charges moving in the presence of the laser field and of the internal Coulomb field. In atoms, within the single active electron (SAE) scheme, a cutoff that can be as large as $300 \omega_{L}$ suggests the possibility of using the generation of high harmonics as a source of far ultraviolet radiation. Recently the study of high order harmonic emission from a molecule has undergone a revival of interest; in fact it has been shown that the emitted field carries information that can be used to observe in real time the behaviour of the molecular charges $[1,2,3]$ or to obtain a tomographic image of the wave function of the molecule $[4,5]$.

This paper is devoted to an analytical and numerical study of the dynamics of a homonuclear two-center molecular ion within the SAE scheme driven by a laser field.

\section{Theory}

We treat the laser as a monochromatic classical electromagnetic field and its interaction with the molecule in the dipole approximation: these are realistic assumptions if the laser pulse has a long duration and has a wavelength comparable 
with that of the visible light. The molecule is supposed to have only one active electron and, because of the large difference between the masses of the nuclei and of the electron, the study of the system is simplified by the ansatz that the motion of the nuclei can be safely described by the classical Newton laws $[6,7,8]$. Finally we analyze only one-dimensional model systems to avoid lengthy numerical calculations.

Let $R_{1}(t)$ and $R_{2}(t)$ be the coordinate of the two nuclei of mass $\mathrm{M}$, the evolution of the state $\psi(x, t)$ of the electron is described by the time dependent Schrödinger equation (TDSE). With these assumptions in mind the equations of motion for the $\psi(x, t)$ and $R_{i}(t)$ are

$$
\begin{aligned}
i \hbar \frac{\partial}{\partial t} \psi(x, t) & =\left[\frac{P^{2}}{2 m}+V(t ; x)+V_{L M}(t)\right] \psi(x, t) \\
M \ddot{R}_{i}(t) & =F_{i e}(t)+F_{i j}(t)+e \mathcal{E}_{0} f(t) \sin \left(\omega_{L} t\right) .
\end{aligned}
$$

The electron-nuclei interaction energy has been taken of the soft-core form

$$
V(t ; x)=-\frac{e^{2}}{\sqrt{\left(x-R_{1}(t)\right)^{2}+x_{0}^{2}}}-\frac{e^{2}}{\sqrt{\left(x+R_{2}(t)\right)^{2}+x_{0}^{2}}} .
$$

$x_{0}$ is a screen factor that eliminate the Coulomb divergence and is chosen as $x_{0}=a_{0} / \sqrt{2}, a_{0}$ is the Bohr radius. The laser-molecule interaction energy is taken as $V_{L M}(t)=-e x \mathcal{E}_{0} f(t) \sin \left(\omega_{L} t\right)$ where $\mathcal{E}_{0}$ is the value of the maximum laser field. The pulse shape $f(t)$ ramps linearly up and down for 2 optical cycles (oc) and remains constant in between; the total pulse duration is 256 oc. The laser wavelength is chosen to be $\lambda_{L}=780 \cdot 10^{-7} \mathrm{~cm}$. Two intensities of the laser field are used: $I_{1}=0.7 \cdot 10^{14} \mathrm{~W} / \mathrm{cm}^{2}$ and $I_{2}=1.61 \cdot 10^{14} \mathrm{~W} / \mathrm{cm}^{2}$. The mass of the nucleus is $M=20 m_{p}$ with $m_{p}$ the proton mass. The forces in the Newton equations for the nuclei are

$$
F_{i j}(t)=e^{2} \frac{R_{i}(t)-R_{j}(t)}{\left[\beta^{2}+\left(R_{i}(t)-R_{j}(t)\right)^{2}\right]^{3 / 2}}
$$

and

$$
F_{i e}(t)=-e^{2} \int \frac{R_{i}(t)-x}{\left[a_{0}^{2}+\left(R_{i}(t)-x\right)^{2}\right]^{3 / 2}}|\psi(x, t)|^{2} d x
$$

$\left[\beta=0.03 a_{0}\right]$. The coupled equations (1) are solved by using a Crank-Nicolson integration code within a spatial box $512 a_{0}$ wide with a grid step of $0.125 a_{0}$. To prevent reflection of the wavefunction at the edge of the box, we use a smooth absorber function to force the electron density to zero at the borders of the grid. The presence of the absorber does not preserve the normalization of the wavefunction and indicates the ionization.

In Fig. 1 and 2 we show an overall view of the dynamics of the molecule with mobile nuclei for the two laser intensities; the initial distance of the two nuclei is $5 a_{0}$. During the operation of the lower intensity laser, almost no ionization of the molecule is present. We see that the nuclei begin a very slow oscillation motion with amplitude $a_{0}$; the center of the oscillation is around $R=6 a_{0}$ and the 

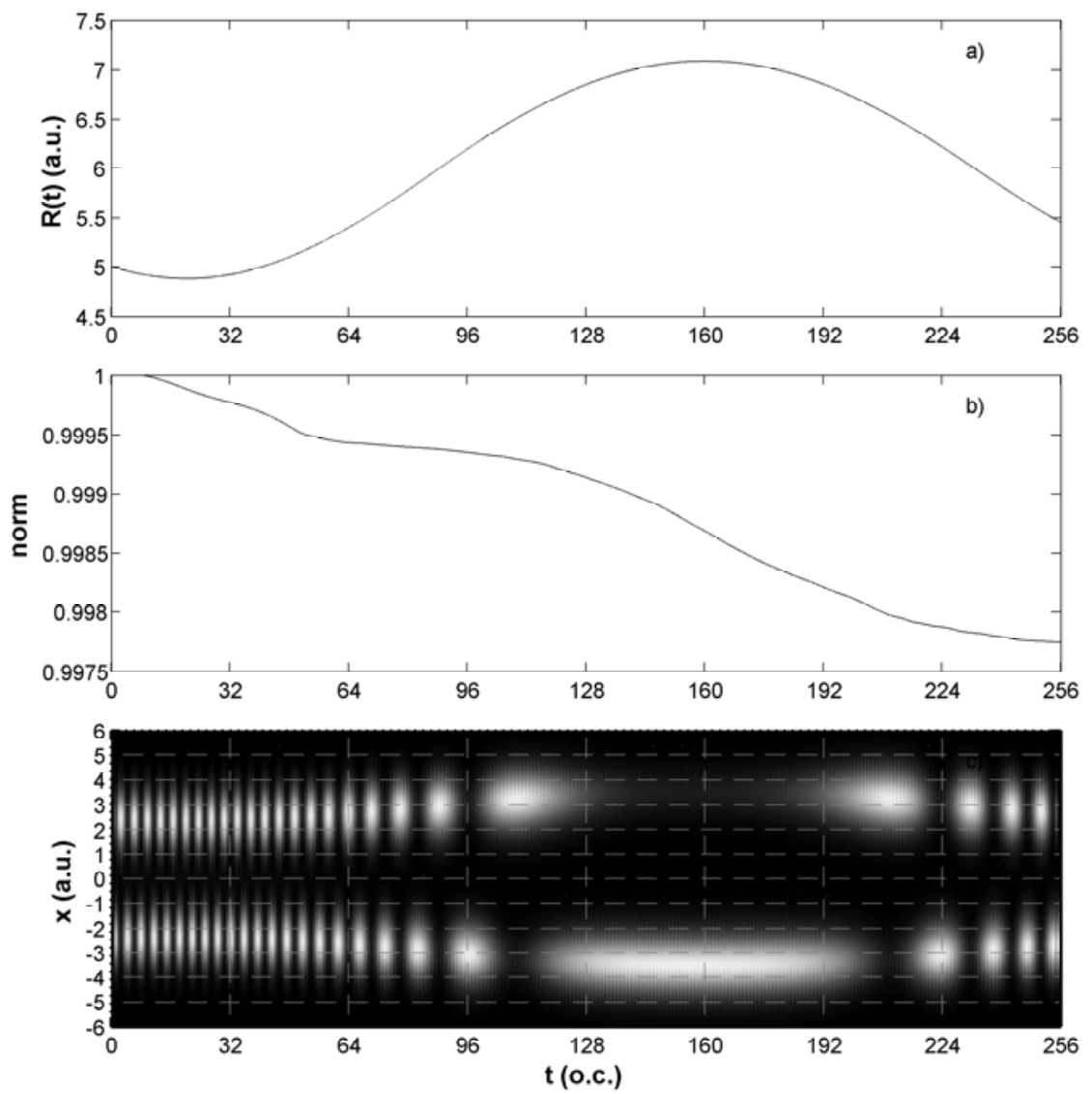

Fig. 1: Global view of the temporal behaviour of the molecule acted upon by the laser. a) Internuclear distance $R$ (in unit of the Bohr radius $a_{0}$ ) vs $t$ in oc. b) Norm $\int_{V}|\psi(x, t)|^{2} d x$ ( $V$ is here the full spatial integration box) of the wavefunction showing that almost no ionization is present during the laser operation. c) $|\psi(x, t)|^{2}$. In this set of calculations the initial nuclear separation is $R=5 a_{0}$ while the initial electron state is the ground bare state. The physical parameters entering the calculations are: laser intensity $I_{1}=0.7 \cdot 10^{14} \mathrm{~W} / \mathrm{cm}^{2}$, laser photon energy $\hbar \omega=1.6 \mathrm{eV}$. The maximum elongation of the internuclear distance is $7.1 a_{0}$.

maximum elongation of the molecular distance is $R=7.1 a_{0}$. The electron wave function, initially in the ground state, oscillates between the two nuclei; at the maximum elongation of the nuclear distance the electron wave function remains trapped over one of the nuclei for more than $30 \mathrm{oc}$. The motion of the nuclei is always very slow and the adiabatic approximation is applicable particularly for $R \approx 7 a_{0}$. This indicates that electron trapping is compatible with mobile nuclei and also that electron ionization is always very small although during the trapping 

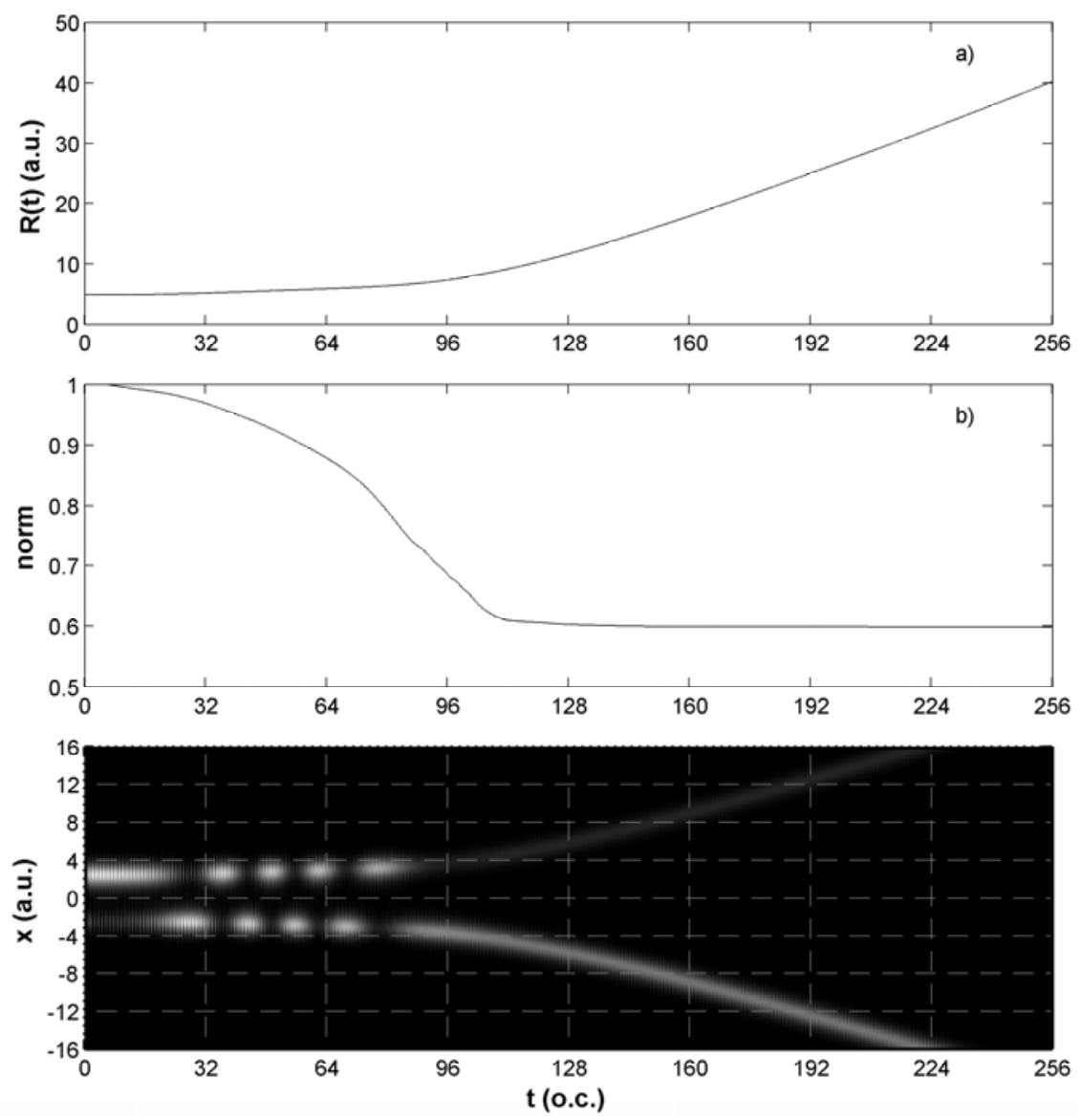

Fig. 2: Global view of the temporal behaviour of the molecule acted upon by the laser. a) Internuclear distance $R$ (in unit of the Bohr radius $a_{0}$ ) vs $t$ in oc. b) Norm $\int_{V}|\psi(x, t)|^{2} d x$ ( $V$ is here the full spatial integration box) showing that almost no ionization is present during the laser operation. c) $|\psi(x, t)|^{2}$. In this set of calculations the initial nuclear separation is $R=5 a_{0}$ while the electron state is the ground bare state. The physical parameters entering the calculations are: laser intensity $I_{2}=1.61 \cdot 10^{14} \mathrm{~W} / \mathrm{cm}^{2}$, laser photon energy $\hbar \omega=1.6 \mathrm{eV}$.

its slope is maximum; all of this seems to disagree with existing models stating that enhanced ionization is achieved during electron localization over a nucleus. In case of stronger laser intensity $I_{2}$ as in Fig. 2, during the first portion of the laser operation, a 30 oc trapping is visible; after few slow electron oscillations, however ionization begins to dominate the molecular dynamics and ignites the Coulomb explosion of the molecule. Very interestingly, after dissociation the dynamics is such as to leave one nucleus stripped of electronic charge while the other 
nucleus remains with more than $60 \%$ of the electron probability density; no further laser induced electron extraction from the remnants is visible. Again the trapping condition can be achieved for a considerably long time.

\subsection{FIXED NUCLEI}

The previous numerical results and their discussion suggests that a large amount of information on the molecular behaviour can be gained by exploiting the simplified fixed nuclei model; we shall address this question here. We shall see also that valuable information can be obtained by the further simplification of considering the molecule as a two-state quantum system.

In the fixed nuclei approximation the evolution of the electron is obtained by numerically solving the TDSE equation

$$
i \hbar \frac{\partial}{\partial t} \psi(x, t)=\left[\frac{P^{2}}{2 m}+V(0 ; x)+V_{L M}(t)\right] \psi(x, t),
$$

considering the nuclei fixed at their initial positions.

To obtain some insight in the dynamics predicted by (5) we make a two state approximation, i.e. we write

$$
|\psi(t)\rangle \simeq b_{0}(t)|0\rangle+b_{1}(t)|1\rangle
$$

$|0\rangle$ and $|1\rangle$ are the ground and first excited eigenstates of the hamiltonian $\hat{H}_{B}=$ $P^{2} / 2 m+V(0 ; x)$. The equations for the two probability amplitudes $b_{i}(t)$ are readily written upon substitution in the Schrödinger equation (5). However we are particularly interested in the possibility of trapping the electron over one of the two nuclei for a long period of time with respect to the laser period $T_{L}[9,10,11]$. We introduce, therefore, the two states (which are not eigenstates of $\hat{H}_{B}$ )

$$
\left\{\begin{array}{l}
|L\rangle=\frac{1}{\sqrt{2}}(|0\rangle+|1\rangle) \\
|R\rangle=\frac{1}{\sqrt{2}}(|0\rangle-|1\rangle)
\end{array}\right.
$$

corresponding to states peaked over one of the two nuclei. $|\psi(t)\rangle$ can be written as a linear combination of these two states: $|\psi(t)\rangle \simeq c_{L}(t)|L\rangle+c_{R}(t)|R\rangle$ with $c_{L}(t)=\left[b_{0}(t)+b_{1}(t)\right] / \sqrt{2}$ and $c_{R}(t)=\left[b_{0}(t)-b_{1}(t)\right] / \sqrt{2}$. The equation for the $c_{i}(t)$ can be profitably written in matricial form as

$$
i \dot{\boldsymbol{c}}=\left\{-\frac{1}{2} \omega_{G} \widehat{\sigma}_{1}+\omega(t) \widehat{\sigma}_{3}\right\} \boldsymbol{c}
$$

where $\boldsymbol{c}(t) \equiv\left(c_{L}, c_{R}\right), \hbar \omega_{G} \equiv E_{1}-E_{0}$ is the energy gap between the two lowest states, $\widehat{\sigma}_{k}$ are the three standard Pauli matrices and, finally, $\hbar \omega(t) \equiv\left\langle 0\left|V_{L M}\right| 1\right\rangle$ is real.

We now suppose that the monochromatic laser field is of the form $\omega(t)=$ 
$\omega_{V} \sin \omega_{L} t$ and we introduce the adimensional quantities

$$
\left\{\begin{aligned}
\tau & \equiv \omega_{L} t \\
\xi & \equiv \frac{\omega_{G}}{\omega_{L}} \\
\Delta & =\frac{\omega_{V}}{\omega_{L}}
\end{aligned}\right.
$$

to change (8) into

$$
i \boldsymbol{c}^{\prime}=\left\{-\frac{1}{2} \xi \widehat{\sigma}_{1}+\Delta \sin \tau \widehat{\sigma}_{3}\right\} \boldsymbol{c},
$$

where the prime means derivative with respect to $\tau$. In addition we define $f(\tau)=$ $\Delta(\cos \tau-1)$ and the operator $\widehat{S}_{0}(\tau)=\exp \left\{i f(\tau) \widehat{\sigma}_{3}\right\}$ solution of

$$
i \widehat{S}_{0}^{\prime}(\tau)=\Delta \sin \tau \widehat{\sigma}_{3} \widehat{S}_{0}
$$

with $\widehat{S}_{0}(0)=\widehat{\mathbb{1}}$. We write $\boldsymbol{c}(\tau)=\widehat{S}_{0}(\tau) \boldsymbol{c}_{1}$. Upon substitution into (10) we obtain the equation for the new unknown $\boldsymbol{c}_{1}$ :

$$
i \boldsymbol{c}_{1}^{\prime}=-\frac{1}{2} \xi\left\{\cos (2 f(\tau)) \widehat{\sigma}_{1}+\sin (2 f(\tau)) \widehat{\sigma}_{2}\right\} \boldsymbol{c}_{1} .
$$

With the help of the Jacobi relations we get

$$
\begin{aligned}
\cos (2 f(\tau))= & \cos (2 \Delta)\left\{J_{0}(2 \Delta)+2 \sum_{k=1}^{\infty}(-1)^{k} J_{2 k}(2 \Delta) \cos (2 k \tau)\right\} \\
& +\sin (2 \Delta) 2 \sum_{k=1}^{\infty}(-1)^{k} J_{2 k+1}(2 \Delta) \cos ((2 k+1) \tau) \\
\sin (2 f(\tau))= & \cos (2 \Delta) 2 \sum_{k=0}^{\infty}(-1)^{k} J_{2 k+1}(2 \Delta) \cos ((2 k+1) \tau) \\
& -\sin (2 \Delta)\left\{J_{0}(2 \Delta)+2 \sum_{k=1}^{\infty}(-1)^{k} J_{2 k}(2 \Delta) \cos (2 k \tau)\right\} .
\end{aligned}
$$

Thus formal integration of (12) gives

$$
i \boldsymbol{c}_{1}=-\frac{1}{2} \xi \int_{0}^{\tau}\left\{\cos \left(2 f\left(\tau^{\prime}\right)\right) \hat{\sigma}_{1}+\sin \left(2 f\left(\tau^{\prime}\right)\right) \hat{\sigma}_{2}\right\} \boldsymbol{c}_{1}\left(\tau^{\prime}\right) d \tau^{\prime}
$$

after integration fast oscillating terms average to zero and the equation for $\mathbf{c}_{1}$ is thus cast into a form suitable for immediate simplification [12-15]; therefore, disregarding the fast oscillating terms in (13) and (14) we get

$$
\left\{\begin{array}{l}
\cos (2 f(\tau)) \approx \cos (2 \Delta) J_{0}(2 \Delta) \\
\sin (2 f(\tau)) \approx-\sin (2 \Delta) J_{0}(2 \Delta)
\end{array}\right.
$$


and our differential equation becomes

$$
i \boldsymbol{c}_{1}^{\prime}=-\gamma \widehat{M} \boldsymbol{c}_{1}
$$

with $\gamma=\frac{1}{2} \xi J_{0}(2 \Delta)$ and $\widehat{M}=\cos (2 \Delta) \widehat{\sigma}_{1}-\sin (2 \Delta) \widehat{\sigma}_{2}$, whose explicit solution is

$$
\boldsymbol{c}_{1}(\tau)=\exp [i \gamma \widehat{M} \tau] \boldsymbol{c}(0)=\widehat{S}_{1}(\tau) \boldsymbol{c}(0)
$$

with $\widehat{S}_{1}(\tau)=[\cos (\gamma \tau) \widehat{\mathbb{1}}+i \sin (\gamma \tau) \widehat{M}]$

The final result for the vector $\boldsymbol{c}(\tau)$ is

$$
\boldsymbol{c}(\tau)=\widehat{S}_{0}(\tau) \widehat{S}_{1}(\tau) \boldsymbol{c}_{0}=\left(\begin{array}{c}
e^{i f(\tau)}\left[c_{L}(0) \cos (\gamma \tau)+i c_{R}(0) e^{2 i \Delta} \sin (\gamma \tau)\right] \\
e^{-i f(\tau)}\left[c_{R}(0) \cos (\gamma \tau)+i c_{L}(0) e^{-2 i \Delta} \sin (\gamma \tau)\right]
\end{array}\right) .
$$

In the particular case that the molecule begins its laser driven-evolution from the ground state $c_{L}(0)=c_{R}(0)=1 / \sqrt{2}$ we have

$$
\left|c_{L}(t)\right|^{2}=\frac{1}{2}\left\{1-\sin \left(\frac{2 \omega_{V}}{\omega_{L}}\right) \sin \left[J_{0}\left(\frac{2 \omega_{V}}{\omega_{L}}\right) \omega_{G} t\right]\right\}
$$

The consequences of this result are clear: when $2 \omega_{V} / \omega_{L}$ is close to a zero of the Bessel function, then the population does not evolve. Essentially formulae (16) correspond to a rotating wave approximation (RWA).

In Fig. 3 we show the temporal evolution of the electron population obtained when the laser intensity is $I_{2}$ for the three cases: moving nuclei (a) (already shown in Fig. 2), fixed nuclei (b) and two-state approximation (c) $\left(\left|c_{L}(t)\right|^{2}\right.$ in (20)); in all cases the initial nuclear distance is $\left|R_{2}(0)-R_{1}(0)\right|=5 a_{0}$ and $|\psi(t=0)\rangle=|L\rangle$. The physical parameters used have been chosen to make $2 \omega_{V} / \omega_{L}$ close to a zero of $J_{0}$. During the first 30 oc the electron density behaves similarly in the three cases. This corresponds to electron trapping condition. At later times only the dynamics of cases (b) and (c) remains apparently identical. Huge differences are present in the emitted spectrum shown in the same figure. The spectrum in part (c) (case of two state approximation) contains very few lines, while the spectra in (a) and (b), present a paradigmatic wide plateau and a cutoff. Even order harmonics are present; their presence can be explained by considering that the electron spends a long time in the neighbourhood of a nucleus and thus experiences a local non symmetric potential. A huge transfer of population does not yield a proper plateau; small but fast oscillations are evidently the source of the harmonics.

\section{Conclusion}

We have found that, when driven by a laser field of suitable intensity and frequency, the electron of a diatomic homonuclear molecule oscillates between the nuclei with a period of several optical cycles and with a transfer of electronic population that can be nearly complete. This is robust against molecular vibrations and is an important feature since it suggests the possibility of controlling experimentally 

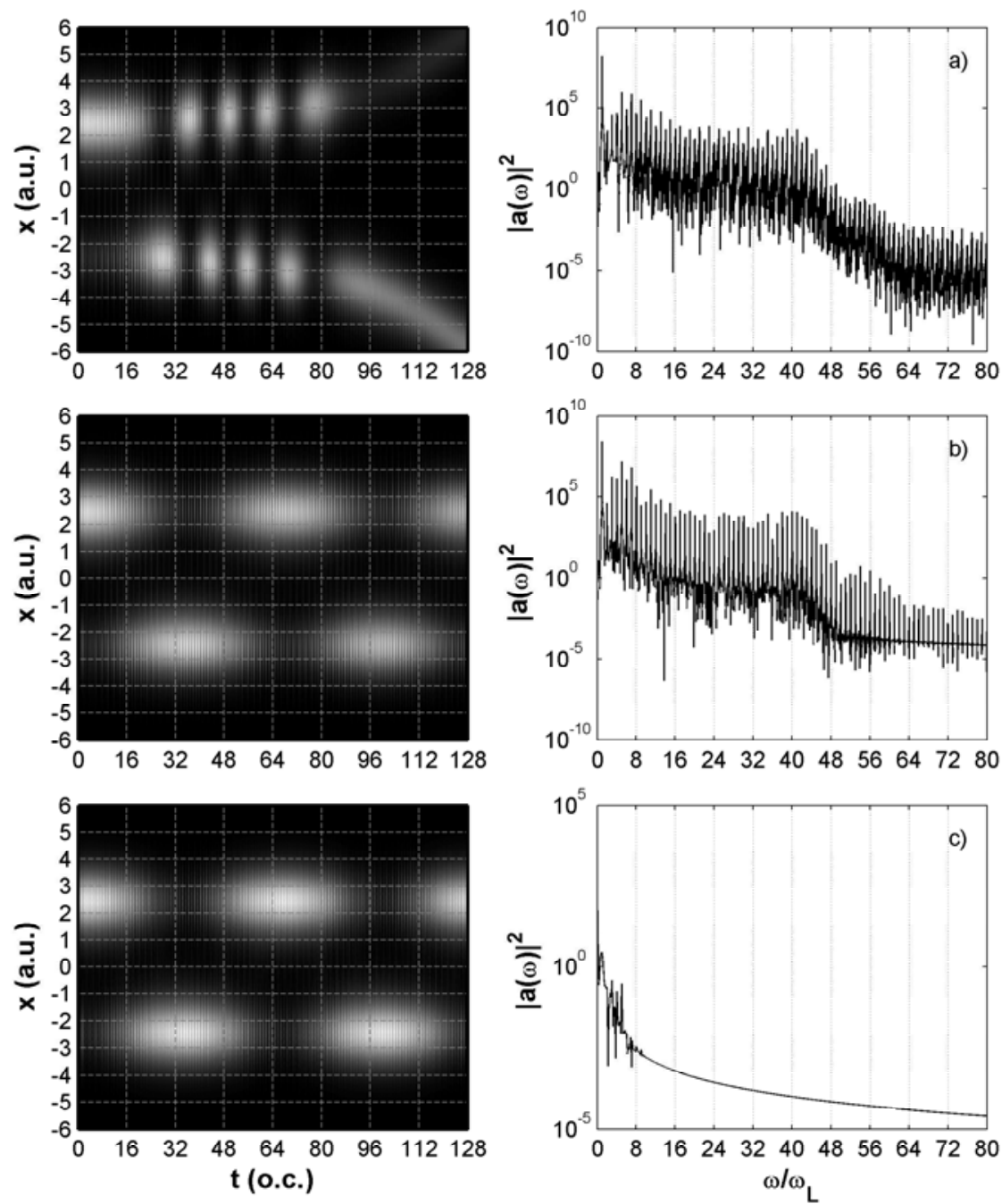

Fig. 3: Plot of $|\psi(x, t)|^{2}$ versus time and spectrum emitted by the molecule. a) Mobile nuclei. b) Fixed nuclei. c) Two-state approximation. The physical parameters entering the calculations are: laser intensity $I_{2}=1.61 \cdot 10^{14} \mathrm{~W} / \mathrm{cm}^{2}$, laser photon energy $\hbar \omega=1.6 \mathrm{eV}$. The initial internuclear distance is $R=5 a_{0}$. 
the migration of the electron between the bound states of the molecule. We have, moreover, compared these results with those obtained by means of a two-level model that is expected to give a good approximation of the dynamics of the system for the chosen laser-atom parameters. We have found that the two-level system can be successfully adopted to describe the behaviour of the electronic population in spite of the fact that it completely fails in reproducing the acceleration of the radiating electron.

\section{Acknowledgments}

This work was supported by the Italian Ministero dell'Istruzione, dell'Università e della Ricerca and in part by Università di Palermo under a PRIN 2004 project.

\section{Bibliography}

[1] E. Fiordilino, R. Daniele and F. Morales, J. Phys. B: Atom. Mol. Opt. Phys. 36, 373 (2003).

[2] G. Castiglia, P. P. Corso, R. Daniele, E. Fiordilino, F. Morales, and F. Persico, J. Mod. Optics 51, 1163 (2004).

[3] P. P. Corso, R. Daniele, E. Fiordilino, J. P. Marangos, F. Morales, and R. Velotta, Phys. Rev. A 40, 053410 (2004).

[4] J. Itatani, J. Levesque, D. Zeidler, H. Niikura, H. Pépin, J. C. Kieffer, P. B. Corkum, and D. M. Villeneuve, Nature 432, 867 (2004).

[5] A. Scrinzi, M. Yu. Ivanov, R. Kienberger, and D. M. Villeneuve, J. Phys. B: At. Mol. Opt. Phys. 39, R1 (2006).

[6] B. Rotenberg, R. Taïeb, V. Véniard, and A. Maquet, J. Phys. B: At. Mol. Opt. Phys. 35, L397 (2002).

[7] R. Daniele, G. Camiolo, G. Castiglia, P. P. Corso, F. Morales, and E. Fiordilino, Appl. Phys. B 78, 813 (2004).

[8] G. Castiglia, G. Camiolo, P. P. Corso, R. Daniele, E. Fiordilino, and F. Morales, Laser Phys. 14, 1185 (2004).

[9] R. Bavli and H. Metiu, Phys. Rev. Lett. 69, 1986 (1992).

[10] M. Grifoni and P. Hanggi, Phys. Rep. 304, 229 (1998).

[11] I. Gilary, N. Moiseyev, S. Rahav and S. Fishman, J. Phys. A Math. Gen. 36 L409 (2003).

[12] J. M. Gomez Llorente and J. Plata, Phys. Rev. A 45, 6958 (1992).

[13] G. S. Agarwal and W. Harshawardhan, Phys. Rev. A 50, 4465 (1994).

[14] A. Santana, J. M. Gomez Llorente and V. Delgado, J. Phys. B At. Mol. Opt. Phys. 342371 (2001)

[15] M. Frasca, Phys. Rev. B 68, 165315 (2003). 